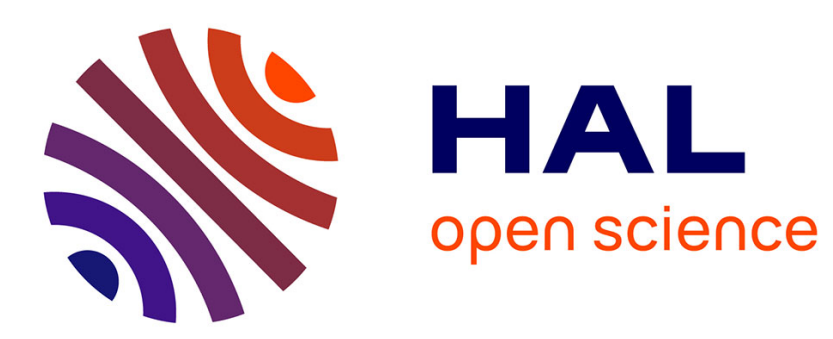

\title{
Efficient Probabilistic Information Broadcast Algorithm over Random Geometric Topologies
}

\author{
Ruijing $\mathrm{Hu}$
}

\section{To cite this version:}

Ruijing Hu. Efficient Probabilistic Information Broadcast Algorithm over Random Geometric Topologies. GLOBECOM, Dec 2015, San Diego, United States. hal-01232676

\section{HAL Id: hal-01232676 \\ https://hal.science/hal-01232676}

Submitted on 23 Nov 2015

HAL is a multi-disciplinary open access archive for the deposit and dissemination of scientific research documents, whether they are published or not. The documents may come from teaching and research institutions in France or abroad, or from public or private research centers.
L'archive ouverte pluridisciplinaire HAL, est destinée au dépôt et à la diffusion de documents scientifiques de niveau recherche, publiés ou non, émanant des établissements d'enseignement et de recherche français ou étrangers, des laboratoires publics ou privés. 


\title{
Efficient Probabilistic Information Broadcast Algorithm over Random Geometric Topologies
}

\author{
Ruijing $\mathrm{Hu}$ \\ Université Pierre et Marie Curie, CNRS/INRIA \\ 4 Place Jussieu, Paris, France \\ Email: ruijing.hu@lip6.fr
}

\begin{abstract}
This paper studies reliability of probabilistic gossip algorithms over the random geometric topologies which model ad hoc networks. We propose an efficient algorithm that ensures higher reliability at lower message complexity than the three families of gossip algorithms. Such an improvement is reasonably estimated by our reliability model. The results obtained by OMNET++ simulator confirm the prediction that our algorithm is the best choice for random geometric networks.
\end{abstract}

\section{INTRODUCTION}

Information broadcast is a basic service for many distributed applications in ad hoc networks. A message generated by a source is transferred to all other sites by successive retransmissions, since sites have no direct connection to each other in the network. In particular, even though global information about the topology is hardly available in large-scale system, an efficient broadcast protocol should still be able to provide high reliability and low latency with tolerable message exchanges.

The simplest solution is using a pure flooding protocol [14]. Upon the first reception of a message, every site delivers it to the application and also forwards it to all sites in its retransmission range once. Evidently, this form of dissemination is very wasteful and may give arise to a broadcast storm [21], entailing for instance packet collision and loss.

A common alternative to the flooding is to require the sites of the system to relay the message only to some of their neighbors. The decision of the neighbors is either carried out in a deterministic or probabilistic way. The problem of the former ensuring high reliability is that its implementation is commonly very hard (i.e., in some schemes it is proven to be NP-hard to reach the optimal performance) and requires more information even if the mobility is not considered [19]. Hence, in our study we are interested in probabilistic schemes that highlight their simplicity and scalability [8], [27]. More precisely, three basic probabilistic gossip algorithms are widely-deployed in ad hoc networks without overlay construction for reliable dissemination: (1) Probabilistic Edge Gossip (GossipPE) [25], (2) Probabilistic Broadcast Gossip (GossipPB) [10], and (3) Probabilistic Inverse Self-degree Broadcast Gossip (GossipPISB) [6]. Furthermore, we propose an efficient algorithm: Probabilistic Inverse Neighbor-degree Edge Gossip (GossipPINE), which exploits local information about sites in a one-hop neighborhood. It performs better than the basic gossip algorithms over two kinds of random geo- metric topologies with and without border effects, which are commonly used to analyze the behavior of ad hoc networks.

On the other hand, we introduce a model to explain the best reliability given by our algorithm GossipPINE amongst all gossip algorithms. Interestingly, we find that the reliability of GossipPINE is accurately estimated for both topologies, and not affected by complex feature of the topology such as edge dependency and border effect. In contrary, the reliability of the other algorithms shows a significant variation from one topology to another. We therefore argue that GossipPINE is robust even in networks whose global characteristics are unknown beforehand.

Extensive simulations on top of OMNET++ [1] almost conform to our theoretical results of reliability. The latency, another important metric for broadcasting service, is also evaluated.

The road-map of this paper is organized as follows. An overview of the random networks and the gossip algorithms is given in Sections II and III. Section IV presents our gossip algorithm. The performance metrics are defined in Section $\mathrm{V}$, while we explain our model in Section VI. Section VII evaluates our simulation and related work is discussed in Section VIII. Section IX concludes this paper.

\section{SySTEM TOPOLOGIES}

A network underlying a large-scale dissemination system can be viewed as a bidirectional or undirected graph. It is comprised of $N$ sites $\left\{s_{1}, s_{2}, \cdots, s_{N}\right\}$. The set of sites $s_{j}$, connected to $s_{i}$ (i.e., $s_{i} \sim s_{j}$ ), is called $s_{i}$ 's neighbors, represented by $\Lambda_{i}$ whose cardinal is the degree of $s_{i}$, denoted $V_{i}$. $P(k)$ represents the degree distribution of sites with $k$ neighbors (i.e., the fraction of sites with degree $k$ ) in the graph and $\bar{V}$ is the mean degree $\left(\bar{V}=\sum_{k=0}^{N-1} P(k) \cdot k\right)$. Moreover, another important property of the graph is defined as follows.

Clustering Coefficient, denoted $\mathbf{C}$ of a given random graph, for distinct sites $s_{i}, s_{j}, s_{k}$, is the conditional probability that, given the existence of edges $s_{i} \sim s_{k}$ and $s_{j} \sim s_{k}$, an edge $s_{i} \sim s_{j}$ also exists (i.e., $P_{\text {connect }}\left(s_{i} \sim s_{j} \mid s_{i} \sim s_{k}, s_{j} \sim s_{k}\right)$ ).

Our study focuses on the random geometric graphs [22], which model ad hoc networks in [6], [10], [25].

More precisely, the sites are distributed uniformly at random in a bounded region, which composes a random geometric graph. In this article, such a region is a rectangular plane with 
length $a$ and width $b$. Furthermore, two sites are connected, as long as the distance between them is at most $\rho$. Based on [23], we can fine-tune $\rho>\sqrt{\frac{(1+\varepsilon) \cdot \ln (N) \cdot a \cdot b}{N \cdot \pi}}$ with a positive constant $\varepsilon$ in order to ensure that the graph is connected.

As observed in [12], this random graph can have Border Effect, where the degree of the sites close to boundaries is much smaller than the mean degree. If we ignore it, the topology, denoted $\mathcal{T}(N, \rho)$, follows a Poisson-distribution [16]: $P_{t}(k)=\exp \left(-\bar{V}_{t}\right) \frac{\bar{V}_{t}{ }^{k}}{k !}$ with mean degree $\bar{V}_{t}=\frac{N \cdot \pi \cdot \rho^{2}}{a \cdot b}$. In [3], the edge dependency is calculated as $C=0.5865$.

Otherwise, the sites with low degree near the boundary, which in fact have an important impact on reliability of gossip algorithm, should be taken into account. As shown in [11], the topology with the border effect, denoted $\mathcal{G}(N, \rho)$, has the degree distribution: $P_{g}(k)=P_{t}(k)\left(\frac{(a-2 \rho)(b-2 \rho)}{a b}+\frac{2 a \rho+(b-2 \rho) \rho}{a b} \psi(k)\right)$ where $\psi(k)=\int_{0}^{1} \exp \left(-\bar{V}_{t}(F(x)-1)\right) \cdot F(x)^{k} \mathrm{~d} x$ with $F(x):=$ $\frac{1}{\pi}\left(x \sqrt{1-x^{2}}-\arccos (x)\right)+1$. Trivially, the mean degree can be calculated as $\bar{V}_{g}=\sum_{k=0}^{N-1} P_{g}(k) \cdot k$.

\section{GossiP ALGORITHMS}

Broadcast in large-scale network is commonly studied on the basis of Algorithm 1. Initially, the source sends a message to all of its neighbors (Lines 2 and 3). A site delivers and retransmits a received message provided it has not previously received it; otherwise, the message is discarded. Sites that have received the message at least once are called infected sites.

Algorithm 1: Generic Gossip Algorithm

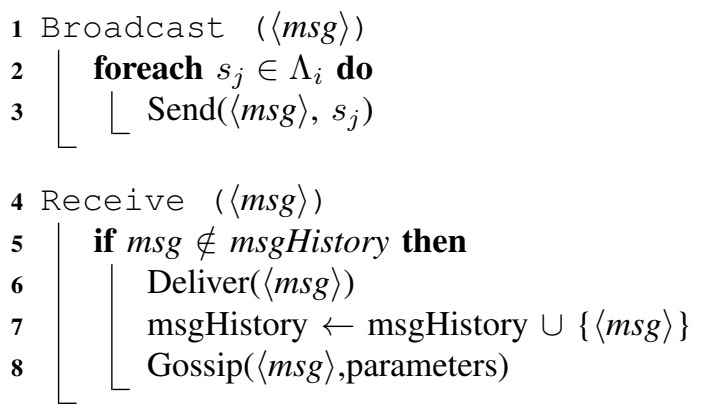

Basically, probabilistic gossip algorithms, which disseminate information in ad hoc networks without overlay construction can be classified into three families, to implement Gossip() procedure: (1) Probabilistic Edge Gossip (GossipPE) [25], (2) Probabilistic Broadcast Gossip (GossipPB) [10], and (3) Probabilistic Inverse Self-degree Broadcast Gossip (GossipPISB) [6]. Besides the received message, the Gossip() procedure takes one parameter which is identical for all sites. In the sequel, Random() generates a random number in the interval $[0,1]$.

Algorithm 2: Probabilistic Edge Gossip (at $s_{i}$ )
$9 / * p_{e}:$ probability to
$\mathbf{1 0}$ GossipPE $\left(\langle m s g\rangle, p_{e}\right)$
\begin{tabular}{l|c}
11 & foreach $s_{j} \in \Lambda_{i}$ do \\
$\mathbf{1 3}$ & if Random ()$\leqslant p_{e}$ then \\
$\operatorname{Send}\left(\langle m s g\rangle, s_{j}\right)$
\end{tabular}

In GossipPE (Algorithm 2), a site $s_{i}$ chooses to send $m s g$ over an edge independently from the other edges with regard to a fixed probability $p_{e}$ (see Line 12$)$. Note that when $p_{e}=1$ for all sites, we obtain the flooding algorithm.

Algorithm 3: Probabilistic Broadcast Gossip (at $s_{i}$ )

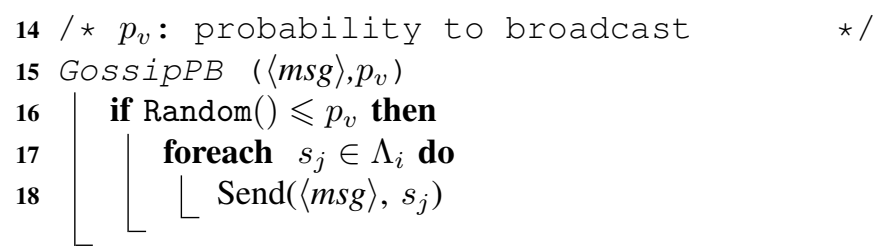

Unlike Algorithm 2, in GossipPB (Algorithm 3), each site, except the source, diffuses $m s g$ to all its neighbors with probability $p_{v}$ (see line 16 ). In particular, when $p_{v}=1$ this protocol becomes the flooding algorithm.

Algorithm 4: Probabilistic Inverse Self-degree Broadcast Gossip (at $s_{i}$ )

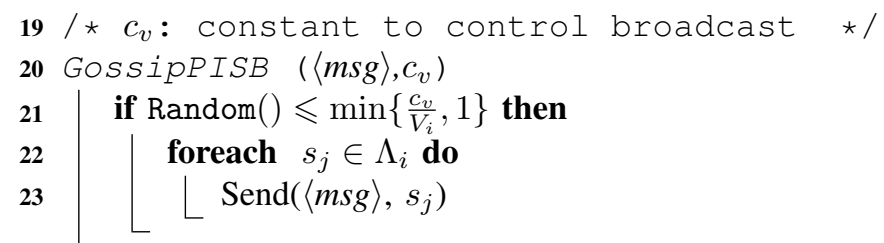

In GossipPISB (Algorithm 4), every site except the source rebroadcasts $m s g$ to all its neighbors independently with probability $\min \left\{\frac{c_{v}}{V_{i}}, 1\right\}$ (see Line 21). When $c_{v} \geqslant$ $\max \left\{V_{1}, V_{2}, \cdots, V_{N}\right\}$ this protocol is the flooding algorithm.

\section{OUR Algorithm}

The idea behind our algorithm is to ensure that sites with very low degree are infected, if one of their neighbors is infected. On the other hand, fewer redundant message copies are received at the sites with high degree. This will be analyzed in Sections VI and VII.

Algorithm 5: Probabilistic Inverse Neighbor-degree Edge Gossip (at $s_{i}$ )

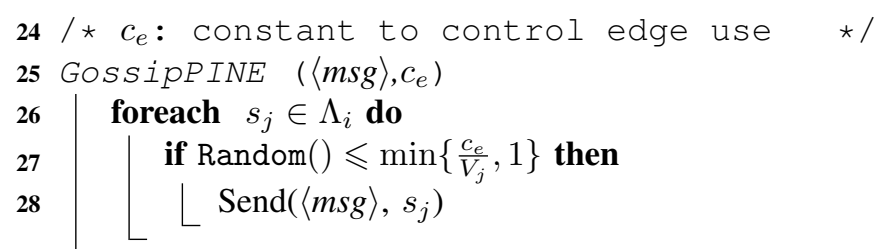

Algorithm 5 shows our Probabilistic Inverse Neighbordegree Edge Gossip, denoted GossipPINE. We assume that every site knows the degree of its neighbors. Like GossipPE, site $s_{i}$ randomly chooses its edges over which $m s g$ should 
be transmitted. However, the probability to send on one edge depends on the degree of the connected neighbor (see Line 27).

\section{Performance Metrics}

For performance evaluation of the gossip algorithms, the following metrics are commonly used [7], [18], [20]:

Message Complexity, denoted $M$ : measures the mean number of messages received (or sent, since no message loss is taken into consideration) by each site: $M=\frac{\Omega}{N-1}$, where $\Omega$ is the total number of messages exchanged during the dissemination.

Reliability, denoted R: is defined as the percentage of messages generated by a source that are delivered by all sites. A reliability value of $100 \%$ is indicative that the algorithm was successful in delivering any given message to all sites (i.e., every site is infected for any given message) ensuring thus atomicity similarly to pure flooding algorithms [15].

Latency, denoted L: measures the number of hops required to deliver a message to all recipients, i.e., the number of hops of the longest path among all the shortest paths from the source to all other sites that received the message.

\section{Reliability Modeling}

In this section, we compare the probabilistic gossip algorithms by our reliability model, which explains the best performance of our algorithm GossipPINE in random geometric graphs.

Since we are only interested in whether a site has been infected by the end of the broadcast, a dissemination graph is exploited for modeling the reliability.

Given a graph $G=(S ; E)$, and a source site $s_{0}$, the probabilistic gossip algorithms follow the same principle: when a new message arrives at a site, this site chooses a subset (possibly empty) of its neighbors in $G$ and forwards the message to these neighbors.

In our analysis, we assume that every site selects a set of neighbors to receive the message in an initial phase. We thus obtain a directed graph $\vec{G}=(S ; \vec{E})$ with sites identical to $G$. For an edge $\left(s_{i} \sim s_{j}\right) \in E$, the arc $\overrightarrow{s_{i} s_{j}}$ is a part of $\vec{G}$, if $s_{i}$ has selected $s_{j}$ in the initial phase. Then, a site $s_{i}$ is infected during dissemination, if there is a path from $s_{0}$ to $s_{i}$ in $\vec{G}$. Furthermore, if every site receives the message, the number of arcs in $\vec{G}$ expresses the message complexity. We call $\vec{G}$

\section{Dissemination Graph.}

We call a site isolated in the dissemination graph, if it has no incoming arcs. Evidently, an isolated site is not infected by a given gossip algorithm. We call the probability that an edge in $E$ becomes an arc towards one site in $S$ the forwarding probability, denoted $p_{\text {forw }}$.

We assume as a precondition that the random network $G=$ $(S ; E)$ is connected. Thus, any message will reach all sites by pure flooding. We model the reliability as the probability that no site is isolated in the dissemination graph. Hence, for a random topology with $N$ sites and degree distribution $P(k)$ we model the probability $R$ as

$$
R=\prod_{i=1}^{N-1}\left(1-\left(1-p_{\text {forw }}(i)\right)^{i}\right)^{P(i) \cdot N}
$$

where $p_{\text {forw }}(i)$ denotes the probability that a site with degree $i$ has an incoming arc from a neighbor. Our evaluation shows that this equation provides a good estimate for the reliability while assuming that every site is isolated independently as done in [8]. However, due to high clustering coefficient in random geometric graphs, one isolated site can even make adjacent sites never receive the message, particularly in sparse density area. Our model shown in Equation (1) will consequently overestimate the reliability, especially in $\mathcal{G}(N, \rho)$ with border effect. Yet, our simulations show that our model still gives a valuable estimate and correctly indicates which algorithm is more or less reliable.

GossipPE enables every site to be selected at random by neighbors with probability $p_{e}$. Therefore, its forwarding probability: $p_{\text {forw }}^{P E}=p_{e}$. In the same way, $p_{\text {forw }}^{P B}=p_{v}$ and $p_{\text {forw }}^{P I N E}(i)=\min \left\{\frac{c_{e}}{i}, 1\right\}$ for GossipPB and GossipPINE respectively. The message reception by GossipPISB in a site $s_{i}$ depends on the degree of its neighbors, while the latter is highly associated with the degree of $s_{i}$. Lemma 1 studies the average degree of an arbitrary neighbor of $s_{i}$ whose degree is given, ignoring the border effect of random geometric graphs.

Lemma 1: In $\mathcal{T}(N, \rho)$, given a site $s_{i}$ with degree $V_{i}$ and one adjacent site $s_{j}$ with $V_{j}$ neighbors, we can get the average value of $V_{j}$ as:

$$
\text { NeighD }=1+\left(V_{i}-1\right) \cdot C+\left(\bar{V}_{t}-1\right) \cdot(1-C)
$$

where $\bar{V}_{t}=\frac{N \cdot \pi \cdot \rho^{2}}{a \cdot b}$ is the mean degree of the graph.

Proof: Figure 1 shows $s_{i}$, a neighbor $s_{j}$, and the retransmission zones (i.e., $\left.\operatorname{Disc}\left(s_{i}\right), \operatorname{Disc}\left(s_{j}\right)\right)$. We call the overlapped part between two discs lune (i.e., Lune $\left(s_{i}, s_{j}\right)$ ). Let disc be the area of $\operatorname{Disc}\left(s_{j}\right)$. Then, the average area of the lune is $C \cdot$ disc. Clearly, the density of sites inside $\operatorname{Disc}\left(s_{i}\right)$, and also inside $\operatorname{Lune}\left(s_{i}, s_{j}\right)$ is $\frac{V_{i}}{d i s c}$. If disc is small, we can assume that the density outside $\operatorname{Disc}\left(s_{i}\right)$ is $\frac{\bar{V}_{t}}{\text { disc }}$. Therefore, $s_{j}$ has $\frac{V_{i}}{\text { disc }} \cdot C \cdot$ disc neighbors in Lune $\left(s_{i}, s_{j}\right), \frac{V}{\text { disc }} \cdot(1-C) \cdot$ disc neighbors outside $\operatorname{Lune}\left(s_{i}, s_{j}\right)$, plus $s_{i}$.

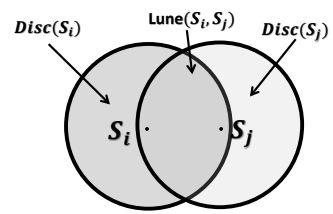

Figure 1: Two kinds of $s_{j}$ 's neighbors

From Lemma 1 , we can derive $p_{\text {forw }}^{\text {PISB }}(i)=$ $\min \left\{\frac{c_{v}}{1+(i-1) \cdot C+\left(\overline{V_{t}}-1\right) \cdot(1-C)}, 1\right\}$ for $\mathcal{T}(N, \rho)$, while we approximate it for $\mathcal{G}(N, \rho)$ in replacing $\bar{V}_{t}$ by $\bar{V}_{g}$ that is the 
mean degree of the random topology with the border effect (see Section II).

Theorem 2: If all algorithms generate the same message complexity, then for $k<\bar{V}$

$$
p_{\text {forw }}^{P I N E}(k)>p_{\text {forw }}^{P I S B}(k)>p_{\text {forw }}^{P E}=p_{\text {forw }}^{P B} .
$$

holds.

Proof: According to the study in [12], we infer input parameters of gossip algorithms to reach a given message complexity. Therefore, when identical message complexity holds for all gossip algorithms, $c_{e}=c_{v}, p_{e}=p_{v}$, and $c_{v} \geqslant p_{e} \cdot \bar{V}$. Trivially, $p_{\text {forw }}^{P E}=p_{\text {forw }}^{P B}$.

Since $k<\bar{V}$ implies $k<N$ eigh $D<\bar{V}$, it follows for $k \geqslant c_{e}: p_{\text {forw }}^{P I N E}=\frac{c_{e}}{k}=\frac{c_{v}}{k}>\frac{c_{v}}{N e i g h D}=p_{\text {forw }}^{P I S B}$, and $\frac{c_{v}}{N e i g h D} \geqslant \frac{p_{e} \cdot \bar{V}}{N e i g h D}>p_{e}=p_{\text {forw }}^{P E}$.Then, $p_{\text {forw }}^{P I N E}(k)>$ $p_{\text {forw }}^{P I S B}(k)>p_{\text {forw }}^{P E}=p_{\text {forw }}^{P B}$.

Furthermore, we can deduce that the sites with degree smaller than $\bar{V}$ are the majority in $\mathcal{T}(N, \rho)$ and $\mathcal{G}(N, \rho)$, which dominate the site isolations. In regard to our reliability model described in Equation (1), GossipPINE, which ensures the message reception in sites with lower degree, is thus more reliable in random geometric graphs than the other gossip algorithms.

\section{PERFormance EVAluAtion}

In this section, we evaluate performance of the four probabilistic gossip algorithms in Sections III and IV: GossipPE, GossipPB, GossipPISB, and our algorithm GossipPINE. These algorithms are executed over two kinds of random geometric graphs described in Section II.

Our simulations are conducted on top of OMNET++. We consider networks composed of $N=1000$ sites and, in order to ensure connectivity, $\varepsilon=1$ for every random geometric graph, where $a=7500, b=3000, \rho=330$, such that the mean degree of $\mathcal{G}(N, \rho)$ (resp., $\mathcal{T}(N, \rho)$ ) is about 14 (resp., 15). The graphs without border effect are generated by bending the rectangular region into a Torus $\mathcal{T}(N, \rho)$, while the Euclidean distance between every site is measured.

The results, obtained by our reliability modeling, are also compared with our simulation. We can observe that in terms of reliability, our algorithm outperforms the other gossip algorithms, which conforms to Theorem 2 .

For each gossip algorithm, we fixed the parameter values to reach a given message complexity in using the method introduced in [12], and then evaluated the reliability and the latency of the algorithm. 200 different messages are generated by 200 different sources that are chosen uniformly amongst 1000 sites over 50 different graphs related to each of the topologies. Then, the results for each message complexity are averaged by the $200 \times 50=10000$ message disseminations.

As a matter of fact, we can configure the topology parameters with other values, whereas the best reliability of our algorithm GossipPINE is always obtained, as estimated by our model for the geometric graphs. Due to lack of space in our paper, we do not give exhaustive presentation.
Reliability: We now discuss the reliability values for the gossip algorithms in $\mathcal{T}(N, \rho)$ and $\mathcal{G}(N, \rho)$, obtained by the simulations as well as our model.

The results are plotted in Figure 2, where the models for GossipPINE and GossipPISB are called ModelPINE and ModelPISB respectively. As explained in Section VI, our model gives the same result for GossipPE and GossipPB. This is depicted as ModelPE.

GossipPINE exhibits the best performance in both Figures 2(a) and 2(b), while GossipPB and GossipPE show the worst reliability. In regard to $\mathcal{T}(N, \rho)$, the reliability is more or less degraded in $\mathcal{G}(N, \rho)$ for every gossip algorithm. Especially, GossipPB is far less reliable than the other algorithms in $\mathcal{G}(N, \rho)$. Compared with $\mathcal{T}(N, \rho)$, on the boundaries of the rectangular field $\mathcal{G}(N, \rho)$ lie a large number of sites with low degree (see Section II). These sites are easily isolated in GossipPB, which results from the clustering effect explained in [12].

Due to the high clustering coefficient, as shown in Lemma 1, sites with low degree are very likely to be clustered together in random geometric graphs (i.e., small groups of interconnected sites with relatively few edges to other groups). As a result, a single isolated site can render many adjacent sites isolated. This effect is further reinforced by the border of the rectangular region comprising sites with low degree. GossipPISB leverage this clustering, so that the forwarding probability $p_{\text {forw }}^{P I S B}(i)$ for small $i$ is larger than $p_{\text {forw }}^{P E}$. It thus mitigates isolating sites in a cluster with low degree and achieve higher reliability than GossipPE.

Since the site isolation dependency is not included in our model, the results from our modeling overestimate the performance obtained by simulation in Figures 2(a) and 2(b). We see that such dependency in $\mathcal{G}(N, \rho)$ has a greater impact on GossipPB and GossipPISB, for which the reliability falls behind the predicted model, in regard with GossipPINE and GossipPE. The reason is that the message retransmitted by GossipPB or GossipPISB stays within a cluster few hops away from the source, when the retransmission probability is not high enough. In accordance with the percolation theory [9], GossipPE is never worse than GossipPB on any topology.

It is worth pointing out that in GossipPINE the probability for a message to be spread out of a cluster with high degree sites and into a cluster with low degree sites is larger than in GossipPISB and GossipPE (see Theorem 2). As expected, GossipPINE is accurately described by our model over both $\mathcal{T}(N, \rho)$ and $\mathcal{G}(N, \rho)$.

Latency: An efficient broadcasting algorithm aims at providing high reliability, while minimizing both message complexity and latency. We analyze the latency performance when the reliability reaches at least $85 \%$. Figures 3(a) and 3(b) show the simulation results in $\mathcal{T}(N, \rho)$ and $\mathcal{G}(N, \rho)$ respectively.

Over all random topologies, after a given message complexity, latency does not decrease anymore, but converges towards the pure flooding approach (i.e., the shortest routes between the source and the other sites), and therefore, towards the minimum latency. 


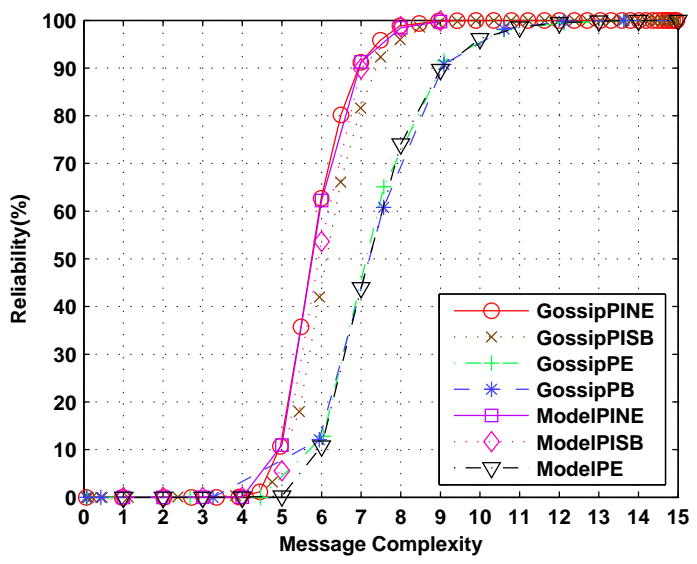

(a) In $\mathcal{T}(N, \rho)$

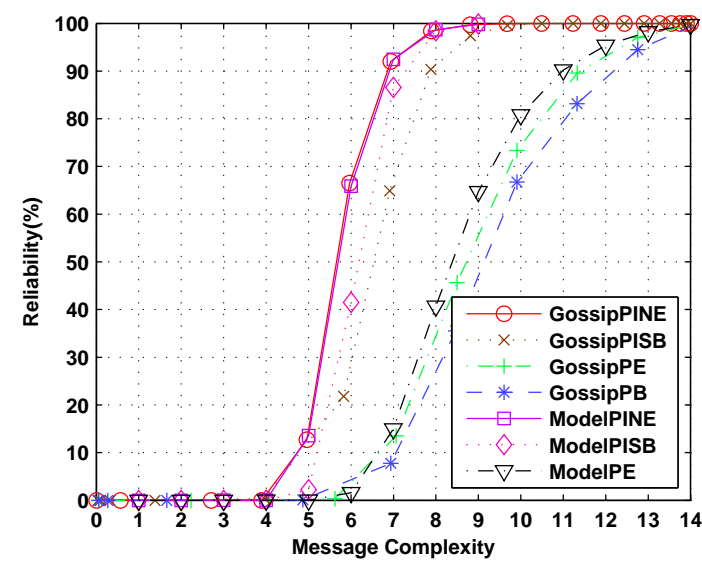

(b) In $\mathcal{G}(N, \rho)$

Figure 2: Reliability comparison of gossip algorithms

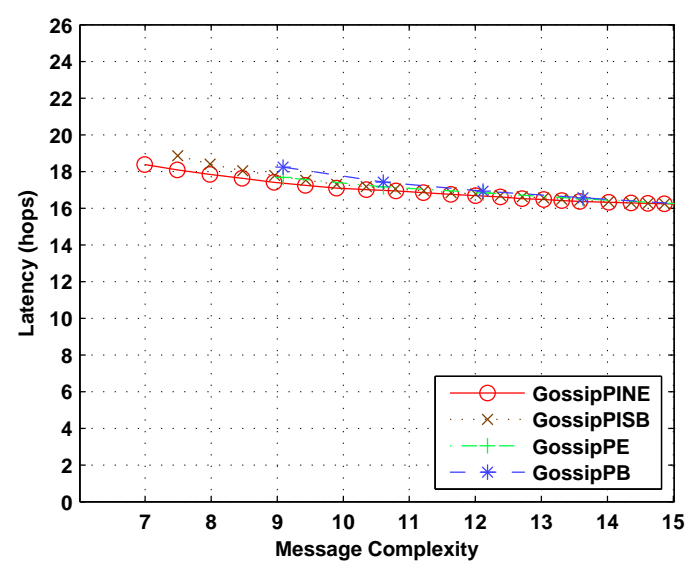

(a) In $\mathcal{T}(N, \rho)$

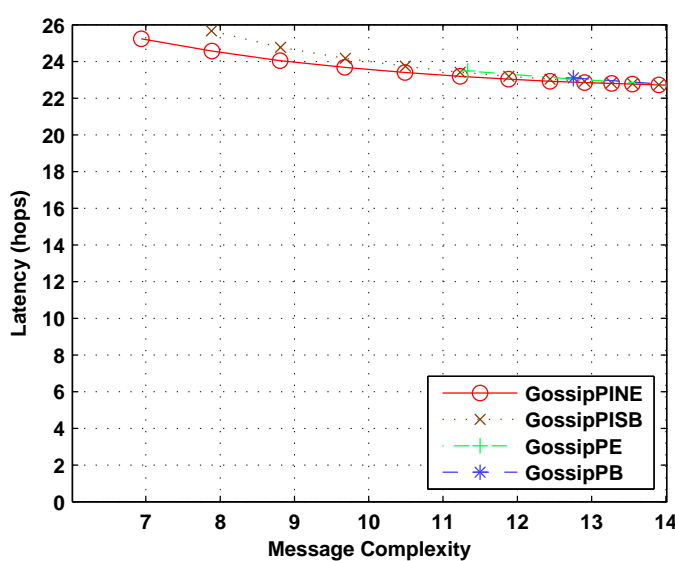

(b) In $\mathcal{G}(N, \rho)$

Figure 3: Latency comparison of gossip algorithms

The latency of our algorithm GossipPINE reaches a lower value in $\mathcal{T}(N, \rho)$ with the smallest message complexity amongst all gossip algorithms. A similar behavior of the latency curves is observed in $\mathcal{G}(N, \rho)$, except that the minimum latency value lies around 22 hops which is larger than in $\mathcal{G}(N, \rho)$, since the diameter of $\mathcal{G}(N, \rho)$ is greater than that of $\mathcal{T}(N, \rho)$.

\section{RELATED WORK}

The initial study on the probabilistic information broadcast in ad hoc networks was conducted in [10] and later implemented on DES-Testbed [4], which evaluates GossipPB and its variants. A bimodal behavior of the reliability has been heuristically analyzed with regard to the retransmission probability $p_{v}$. More precisely, from a threshold value of $p_{v}$, all the sites can receive almost all the messages. Otherwise, the message can rarely reach all sites in the system. In [24], such a threshold is given on the basis of percolation theory. The percolation driven flood routing algorithm proposed in
[28] can be seen as an advanced version of GossipPB in largescale ad hoc networks. It gives an answer how to choose $p_{v}$ to ensure high reliability. In Section VI, we model the reliability as a function of the $p_{v}$, which at the same time takes into account the properties of underlying networks, such as degree distribution. While assuming the site isolation independency in the end of the dissemination, our model accurately establishes such a relation in infinite random geometric graphs. Even in the graphs with border effect, reasonable estimation can be adaptively given by the corrective degree distribution. Furthermore, it explains the higher reliability obtained by our algorithm than the other gossip algorithms.

GossipPISB proposed in [6] tailors the retransmission probability to the local topology, where sites in sparse density area retransmit with high probability. We have shown that the sites around boundaries of the region are hardly infected when small message complexity is allowed. In [8], the authors model the reliability, and improves the protocol by adding a pull phase. But pull algorithms are difficult to carry out 
[15]. Similarly, the authors in [2] distinguish all sites by four levels. The probability in each level is reversely proportional to the number of levels predefined in ad hoc network. Nonetheless, no theoretical analysis are proposed. The Smart Gossip protocol in [17] constructs local relationship trees in two-hop neighborhoods, which consist of a parent, children and siblings. Every parent decides on an optimal forwarding probability that is computed by its children under reliability and topology constraint. Maintenance of the relationship trees is required. Moreover, in [5], the authors specify forwarding probability of a site in ad-hoc networks as function of both its degree and the mean degree of its neighbors, while introducing a mechanism where if a site suspects that some of its neighbors have not received the message, it rebroadcasts the message regardless of the initial decision. The reliability is heuristically studied. In contrary, our algorithm is efficient and very simple to apply in random geometric networks, which is confirmed by both our model and simulation.

As explained in [12] that GossipPE is never worse than GossipPB in any topology, the directional antenna is applied to implement GossipPE in ad hoc network in [25], which shows a better performance than GossipPB. In comparison, our algorithm GossipPINE is robust against border effect that is a common characteristic in the realistic environment with long boundary.

Other probabilistic approaches include counter-based [21], distance-based [13], and location-based mechanisms [26]. In regard to $\operatorname{Gossip} P B$, variant protocols arm a time counter as shown in [21] for copy accumulations. Hop Counter Aided Broadcast (HCAB) protocol in [13] makes the sites, upon the first reception of a message, start a random timer and record the value of hop counter. The message will be relayed by the site if, when the timer expires, no message with a hop counter higher than the first was received. Implicitly, every site in HCAB attempts to know whether each relay is done by some other sites, hopefully covering (i.e., infecting) additional regions of the network. Another distance-based protocol is also proposed in [13], which is called Self-Adaptive Probability Broadcasting (SAPB) protocol. It stipulates that the probability of a site to relay a message depends on coverage (i.e., infection) contribution brought by this site. However, those works contain little theoretical evaluation of the schemes.

\section{CONCLUSION}

In this article, we have proposed an efficient probabilistic broadcast algorithm and reliability model to compare probabilistic gossip algorithms in random geometric networks. Our model gives a reasonable estimation, and explains the best reliability obtained by our gossip algorithm GossipPINE, which is confirmed by our simulations. GossipPINE also shows desirable latency performance for high reliability. In particular, our algorithm has the best resilience to the border effect degrading the reliability.

As a near future work, the random mobility in the network will be evaluated and studied.

\section{REFERENCES}

[1] Omnet++: Discrete event simulation system http://www.omnetpp.org/.

[2] J.-D. Abdulai, A. Mohammed, K. S. Nokoe, and E. Oyetunji. Route discovery in wireless mobile ad hoc networks with adjusted probabilistic flooding. adaptive science technology. ICAST, 2009.:99-109, 2009.

[3] C. Avin. Distance graphs: From random geometric graphs to bernoulli graphs and between. DIALM-POMC, pages 71-78, 2008.

[4] B. Blywis, M. Günes, F. Juraschek, and S. Hofmann. Gossip routing in wireless mesh networks. ISPIMRC, pages 1572-1577, 2010.

[5] J. Cartigny and D. Simplot. Border node retransmission based probalistic broadcast protocols in ad-hoc networks. Annual Hawaii International Conference on System Sciences, pages 6-9, Jan. 2003.

[6] V. Drabkin, R. Friedman, G. Kliot, and M. Segal. Rapid: Reliable probabilistic dissemination in wireless ad-hoc networks. Technical report, Computer Science Department, Technion - Israel Institute of Technology, 2006

[7] P. T. Eugster, R. Guerraoui, and P. Kouznetsov. $\delta$-reliability: A probabilistic measure of broadcast reliability. International Conference on Distributed Computing Systems, pages 24-26, Mar. 2004.

[8] R. Friedman, V. Drabkin, G. Kliot, and M. Segal. On reliable dissemination in wireless ad-hoc networks. IEEE Transactions on Dependable and Secure Computing, 8(6):866-882, 2011.

[9] G. Grimmett. Percolation. Springer, 1989.

[10] Z. Haas, J. Halpern, and L. Li. Gossip-based ad hoc routing. INFOCOM, 3:1707-1716, 2002.

[11] R. Hu. Epidemic dissemination algorithms in large-scale networks: comparison and adaption to topologies. $\mathrm{PhD}$ thesis, Université de Pierre Marie Curie, Dec. 2013.

[12] R. Hu, J. Sopena, L. Arantes, P. Sens, and I. Demeure. Fair comparison of gossip algorithms over large-scale random topologies. International Symposium on Reliable Distributed Systems, pages 331-340, Oct. 2012.

[13] Q. Huang, Y. Bai, and L. Chen. Efficient lightweight broadcasting protocols for multi-hop ad-hoc networks. International symposium on personal, indoor and mobile radio communications, pages 1-5, 2006.

[14] J. Jetcheva, Y. Hu, D. Maltz, and D. Johnson. A simple protocol for multicast and broadcast in mobile ad hoc networks. Internet Draft: draft-ietf-manet-simple-mbcast-01.txt, 2001.

[15] A.-M. Kermarrec, L. Massoulié, and A. Ganesh. Probabilistic reliable dissemination in large-scale systems. IEEE TPDS, 3:248-258, 2003.

[16] Z. Kong and E. M. Yeh. Characterization of the critical density for percolation in random geometric graphs. ISIT, 2007.

[17] P. Kyasanur, R. R. Choudhury, and I. Gupta. Smart gossip: An adaptive gossip-based broadcasting service for sensor networks. International Conference on Mobile Adhoc and Sensor Systems, pages 91-100, 2006.

[18] J. Leitao, J. Pereira, and L. Rodrigues. Epidemic broadcast trees. SRDS, pages 301-310, 2007

[19] J. Lipman, P. Boustead, and J. Judge. Neighbor aware adaptive power flooding in mobile ad hoc networks. International Journal of Foundations of Computer Science, 14(2):237-252, 2003.

[20] J. Luo, P. T. Eugster, and J.-P. Hubaux. Route driven gossip: Probabilistic reliable multicast in ad-hoc networks. INFOCOM, 3:2229-2239, 2003.

[21] S.-Y. Ni, Y.-C. Tseng, Y.-S.Chen, and J.-P. Sheu. The broadcast storm problem in a mobile ad hoc network. International Conference on Mobile Computing and Networking, 8:151-162, 1999.

[22] M. Penrose. Random Geometric Graphs, volume 5. Oxford University Press, oxford studies in probability edition, May 2003.

[23] T. Philips, S. Panwar, and A. Tantawi. Connectivity properties of a packet radio network model. IEEE Transactions on Information Theory, pages 1044-1047, 1989.

[24] Y. Sasson, D. Cavin, and A. Schiper. Probabilistic broadcast for flooding in wireless mobile ad hoc networks. Wireless Communications and Networking, 2:1124-1130, 2003.

[25] C.-C. Shen, Z. Huang, and C. Jaikaeo. Directional broadcast for mobile ad hoc networks with percolation theory. IEEE Transactions on Mobile Computing, 5(4):317-332, Apr. 2006.

[26] Y.-C. Tseng, S.-Y. Ni, and E.-Y. Shih. Adaptive approaches to relieving broadcast storms in a wireless multihop mobile ad hoc network. Transactions on Computers, pages 545-557, 2003.

[27] D. Ustebay, R. Castro, and M. Rabbat. Efficient decentralized approximation via selective gossip. Journal of Selected Topics in Signal Processing, PP(99):1, 2011.

[28] G. Vakulya and G. Simo. Energy efficient percolation-driven flood routing for large-scale sensor network. IMCSIT, 3:877-883, 2008. 